\title{
Мини-инвазивное многососудистое
} коронарное шунтирование безопасно и эффективно: опыт первых 500 случаев

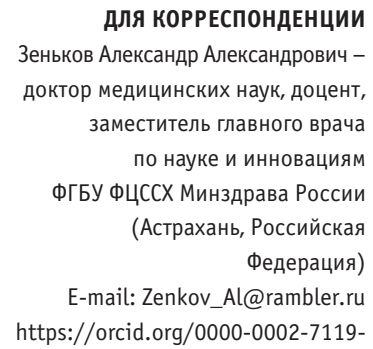

ДЛЯ КОРРЕСПОНДЕНЦИИ Зеньков Александр Александрович доктор медицинских наук, доцент, заместитель главного врача по науке и инновациям ФГБУ ФцсСХ Минздрава России (Астрахань, Российская Федерация)

E-mail: Zenkov_Al@rambler.ru https://orcid.org/0000-0002-7119-

2340

Ключевые слова: ишемическая болезнь сердца, мини-инвазивное коронарное шунтирование, мини-инвазивная реваскуляризация миокарда

\author{
Зеньков А.А. ${ }^{1}{ }^{2}$, Исаев М.Н. ${ }^{1}$, Кондратьев Д.А. ${ }^{1}$, Чернов И.И. ${ }^{1}$, Тарасов Д.Г. ${ }^{1,2}$ \\ ${ }^{1}$ Федеральное государственное бюджетное учреждение «Федеральный центр сердечно-сосудистой хирургии» \\ Министерства здравоохранения Российской Федерации (г. Астрахань), 414011, г. Астрахань, Российская Феде- \\ рация \\ 2 Федеральное государственное бюджетное образовательное учреждение высшего образования «Астраханский \\ государственный медицинский университет» Министерства здравоохранения Российской Федерации, 414000, \\ г. Астрахань, Российская Федерация
}

Инвазивность коронарного шунтирования остается значительной, она не уменьшилась за последние несколько десятков лет. Развитие новых мини-инвазивных технологий в коронарной хирургии основано на стремлении оптимизировать результаты хирургического лечения пациентов с ишемической болезнью сердца (ИБС), особенно при повышенном риске осложнений, связанных с экстракорпоральным кровообращением (ЭКК), стернотомией и манипуляциями на аорте.

Цель работы - провести анализ непосредственных и среднесрочных отдаленных результатов многососудистого мини-инвазивного коронарного шунтирования (МИКШ) в когорте первых 500 пациентов.

Материал и методы. С 2011 по 2021 г. 500 пациентам с ИБС при многососудистом поражении коронарных артерий выполнено МИКШ. Стратегия вмешательства была направлена на выполнение полной реваскуляризации миокарда через левостороннюю мини-торакотомию на работающем сердце. Возраст пациентов - 58,5+7,9 года, в группе было 70 (14,0\%) женщин. Сахарный диабет выявлен у 105 (21,0\%) пациентов, ожирение - у 187 (37,4\%), генерализованный

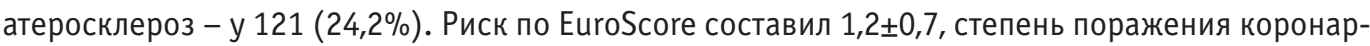
ного русла по SyntaxScore - 26,4 $\pm 8,8$.

Результаты. Средняя продолжительность операции составила 254,5 84,7 мин, число дистальных анастомозов - 2,5ะ0,7. 189 (37,8\%) пациентам выполнено маммаро-аортокоронарное шунтирование, коронарное шунтирование без манипуляций на аорте произведено 311 (62,2\%) пациентам, в том числе полная артериальная реваскуляризация миокарда - 276 (55,2\%) пациентам, гибридная реваскуляризация миокарда - 29 (5,8\%). ЭКК применено у 24 (4,8\%) пациентов, из них экстренная конверсия выполнена у 13 (2,6\%) пациентов. Медиана интраоперационной кровопотери составила $250(200 ; 300)$ мл, кровопотери за 1-е сутки после вмешательства 270 (150; 350) мл. Переливание крови и ее компонентов выполнено 47 (9,4\%) пациентам. Длительность искусственной вентиляции легких в послеоперационном периоде составила $5,0(3,0 ; 8,0)$ ч, время нахождения в отделении реанимации и интенсивной терапии - 19,5 (17,0; $24,0)$ ч. Не наблюдалось случаев глубокой раневой инфекции. Периоперационный инсульт зафиксирован у 2 (0,4\%) пациентов, инфаркт миокарда - у 7 (1,4\%), 30-дневная летальность составила 0,6\% (3 пациента). Длительность послеоперационного пребывания в стационаре составила $7(7 ; 10)$ койко-дней, медиана времени возврата к полной физической активности $14(9 ; 24)$ дней. При длительности наблюдения 4,8 $(1,4 ; 7,3)$ года кумулятивная выживаемость составила 93,4\%, показатель отсутствия неблагоприятных сердечных и мозговых событий $90,7 \%$.

Заключение. МИКШ является безопасным вмешательством, ассоциируясь с низкой частотой периоперационных осложнений, конверсий к ЭКК и стернотомии, короткими длительностью пребывания пациента в стационаре и временем возврата к полной физической активности. МИКШ может быть применено у пациентов с ИБС при многососудистом поражении с сохранением эффективности коронарных вмешательств в среднесрочном отдаленном периоде наблюдения, сравнимой с традиционным АКШ. 
Финансирование. Исследование не имело спонсорской поддержки.

Конфликт интересов. Авторы заявляют об отсутствии конфликта интересов.

Для цитирования: Зеньков А.А., Исаев М.Н., Кондратьев Д.А., Чернов И.И., Тарасов Д.Г. Мини-инвазивное многососудистое коронарное шунтирование безопасно и эффективно: опыт первых 500 случаев // Клиническая и экспериментальная хирургия. Журнал имени академика Б.В. Петровского. 2021. Т. 9, № 4. С. 20-28. D0I: https://doi. org/10.33029/2308-1198-2021-9-4-20-28

Статья поступила в редакцию 01.08.2021. Принята в печать 01.11.2021.

Minimally invasive multivessel coronary artery bypass grafting safe and efficient: the experience of the first 500 cases

Ziankou A.A. ${ }^{1,2}$, Isaev M.N. ${ }^{1}$, Kondratyev D.A. ${ }^{1}$, Chernov I.I. ${ }^{1}$, Tarasov D.G. ${ }^{1,2}$

${ }^{1}$ Federal Center for Cardiovascular Surgery (Astrakhan), 414011, Astrakhan, Russian Federation

${ }^{2}$ Astrakhan State Medical University of the Ministry of Healthcare of the Russian Federation, 414000, Astrakhan, Russian Federation

The invasiveness of coronary artery bypass grafting remains considerable and has not decreased in over several decades. The development of new minimally invasive technologies in coronary surgery is based on the intention to optimise the results of surgical treatment of patients with coronary heart disease (CAD), especially those with increased risk of complications associated with extracorporeal circulation (ECC), sternotomy and aortic manipulations.

Aim - to carry out the analysis of immediate and mid-term results of multivessel minimally invasive coronary artery bypass grafting (MICS CABG) in a cohort of the first five hundred patients.

Material and methods. From 2011 to 2021, 500 CAD patients with CAD at multivessel lesions of the coronary arteries underwent MICS CABG. Operation strategy was directed to perform full myocardial revascularization via left mini-thoracotomy on the beating heart. The age of the patients was $58.5 \pm 7.9$ years, there were $70(14.0 \%)$ women in the group. Diabetes mellitus was detected in $105(21.0 \%)$ patients, obesity - in $187(37.4 \%)$, generalized atherosclerosis - in 121 (24.2\%) patients. The risk for EuroScore was $1.2 \pm 0.7$, the degree of coronary bed damage according to the SyntaxScore was $26.4 \pm 8.8$. Results. The average operation time was $254.5 \pm 84.7$ minutes, the number of distal anastomoses was 2.5 \pm 0.7 . In $189(37.8 \%)$ patients, mammaro-coronary artery bypass grafting was performed, coronary bypass surgery without manipulation on the aorta was performed in $311(62.2 \%)$ patients, including complete arterial myocardial revascularization - $276(55.2 \%)$ patients, hybrid myocardial revascularization - $29(5.8 \%)$ patients. ECC was used in 24 (4.8\%) patients, of which emergency conversion was performed in $13(2.6 \%)$ patients. The median intraoperative blood loss was $250(200 ; 300) \mathrm{ml}$, blood loss for the $1^{\text {st }}$ day after the intervention $-270(150 ; 350) \mathrm{ml}$. Transfusion of blood and its components was performed in $47(9.4 \%)$ patients. The postoperative ventilation time was $5.0(3.0 ; 8.0)$ hours, ICU-stay $19.5(17.0 ; 24.0)$ hours. There were no cases of deep wound infection. Perioperative stroke was recorded in $2(0.4 \%)$ patients, myocardial infarction - in $7(1.4 \%)$, the 30 -day mortality was $0.6 \%$ (3 patients). The postoperative hospital stay was $7(7 ; 10)$ days, median time to return to full physical activity $14(9 ; 24)$ days. With a follow-up duration of $4.8(1.4 ; 7.3)$ years, the cumulative survival rate was $93.4 \%$, the absence of major adverse cardiac and cerebrovascular events was $90.7 \%$.

Conclusion. MICS CABG is a safe operation, associated with a low incidence of perioperative complications, conversions to ECC and sternotomy, short hospital stay, and return time to full physical activity. MICS CABG can be applied to multi-vessel coronary heart disease patients saving the effectiveness during mid-term follow-up, comparable to traditional CABG.

Funding. The study had no sponsor support.

Conflict of interest. The authors declare no conflict of interest.

For citation: Ziankou A.A., Isaev M.N., Kondratyev D.A., Chernov I.I., Tarasov D.G. Minimally invasive multivessel coronary artery bypass grafting safe and efficient: the experience of the first 500 cases. Clinical and Experimental Surgery. Petrovsky Journal. 2021; 9 (4): 20-8. D0I: https://doi.org/10.33029/2308-1198-2021-9-4-20-28 (in Russian)

Received 01.08.2021. Accepted 01.11.2021.
CORRESPONDENCE

Aliaksandr A. Ziankou MD, Docent, Deputy Chief Physician for Science and Innovation, Federal Center for Cardiovascular Surgery (Astrakhan, Russian Federation)

E-mail: Zenkov_Al@rambler.ru https://orcid.org/0000-0002-71192340

\section{Keywords:}

coronary heart disease, minimally invasive coronary artery bypass grafting, minimally invasive myocardial revascularization 
И нвазивность коронарного шунтирования (КШ) остается значительной и не уменьшилась за последние 40-50 лет [1]. Традиционное аортокоронарное шунтирование (АКШ) имеет потенциальную опасность развития ряда осложнений - это посткардиотомный синдром, значимая кровопотеря в периоперационном периоде, гнойно-воспалительные осложнения, диастаз грудины, неврологический дефицит. Также имеются определенные ограничения в реабилитации пациентов, нередко встречаются низкий уровень качества жизни и сомнительный косметический эффект [2]. С учетом частоты и структуры периоперационных осложнений, длительности нахождения в реанимации и стационаре, времени восстановления и возврата к труду лечение с использованием традиционного КШ имеет достаточно высокую стоимость $[3,4]$. Уровень качества жизни в 30-дневный срок после вмешательства и удовлетворенность лечением в течение 6 мес после выписки из стационара значительно выше после чрескожного коронарного вмешательства (ЧКВ) по сравнению с традиционным КШ $[5,6]$.

С целью оптимизации результатов хирургической реваскуляризации миокарда некоторые ее аспекты должны быть улучшены, особенно в эпоху роста и порой злоупотребления ЧКВ. На это направлено развитие новых методов миниинвазивной коронарной хирургии, что в первую очередь важно для пациентов при повышенном риске осложнений, связанных с экстракорпоральным кровообращением (ЭКК), стернотомией и манипуляциями на аорте. Основной целью мини-инвазивной реваскуляризации миокарда (МИРМ) является уменьшение числа прогнозируемых осложнений и ускорение выздоровления пациента при условии сохранения эффективности коронарных операций и длительности лечебного эффекта. В настоящее время мини-инвазивное коронарное шунтирование (МИКШ) в большинстве случаев определяют как шунтирующую операцию, не требующую срединной стернотомии и выполняемую с ЭКК или без него $[7,8]$. На сегодняшний день предложен ряд минимально инвазивных хирургических альтернатив традиционному АКШ, однако каждый из них имеет серьезные недостатки. Так, КШ на работающем сердце выполняется через стернотомию, мини-инвазивный прямой маммарокоронарный анастомоз (MIDCABG) используется только при поражении коронарных артерий передней поверхности сердца, гибридная реваскуляризация миокарда ограничена показаниями к ЧКВ, а робот-ассистированные операции затратны. Стали актуальны разработка и внедрение стратегии МИРМ, которая бы заключала известные позитивные технологии в одной процедуре (мини-инвазивный доступ, работающее сердце, возможность применения артериальной реваскуляризации миокарда, техники no-touch aorta, бимаммарного шунтирования др.), теоретически оптимизируя пропорцию риск/преимущество для коронарной хирургии. В данной статье приведен опыт первых 500 операций МИРМ у пациентов с многососудистым поражением коронарных артерий, который может быть полезен при определении места и перспектив МИКШ в современной коронарной хирургии.

Цель - провести анализ непосредственных и среднесрочных отдаленных результатов многососудистого МИКШ через левостороннюю миниторакотомию на работающем сердце в когорте первых 500 пациентов.

\section{Материал и методы}

В исследование включены 500 пациентов с ишемической болезнью сердца (ИБС) при многососудистом поражении коронарных артерий, которым с 2011 по 2021 г. выполнено МИКШ. Показаниями к МИКШ являлись многососудистое поражение коронарных артерий, т.е. гемодинамически значимый стеноз или окклюзия коронарной артерии диаметром $\geq 1,5$ мм и степенью сужения $\geq 70 \%$ из 2 или 3 бассейнов передней межжелудочковой ветви левой коронарной артерии (ЛКА), огибающей ветви ЛКА или правой коронарной артерии либо гемодинамически значимого стеноза ствола ЛКА; стабильная стенокардия напряжения II-IV функционального класса по Канадской классификации (Canadian Cardiovascular Society, CCS); ранняя постинфарктная стенокардия; безболевая ишемия миокарда. По коронарной анатомии показаниями к МИКШ служили стеноз ствола ЛКА, трехсосудистое поражение, комплексные проксимальные поражения бассейна ЛКА, комплексные поражения задней и боковой поверхности сердца. По сопутствующей патологии показаниями к МИКШ были ожирение, сахарный диабет, хроническая обструктивная болезнь легких, заболевания с длительным использованием кортикостероидов и нарушениями опорно-двигательного аппарата, а также возрастные пациенты и приверженность пациента к МИРМ. Противопоказание к МИКШ - необходимость в экстренной реваскуляризации при остром инфаркте миокарда или остром коронарном синдроме. Потенциальными противопоказаниями к МИКШ выступили КШ в анамнезе, фракция выброса левого желудочка <30\% и билатеральная окклюзия подвздошных артерий.

Стратегия МИКШ была направлена на выполнение полной МИРМ через левостороннюю миниторакотомию на работающем сердце. Выполняли артериальную реваскуляризацию миокарда без манипуляций на аорте или маммаро-аортокоронарное шунтирование (МАКШ). Доступ осуществлялся 
в пятом или шестом межреберье при помощи ретракторной системы ThoraTrak ${ }^{\mathrm{TM}}$ MICS System (Medtronic). Для формирования дистальных анастомозов применялись стандартные и мини-инвазивные системы стабилизации и позиционирования Octopus $^{\circledR}$ Nuvo Tissue Stabilizer и Starfish ${ }^{\circledR}$ NS Heart Positioner (Medtronic, США). Для артериальной реваскуляризации использовали различные кондуиты (правая и левая внутренние грудные артерии, лучевая артерия, правая желудочно-сальниковая артерия) в виде композитно-секвенциальной реконструкции или как in situ графты. Варианты выполнения полной МИРМ без затрагивания аорты представлены предварительно [9]. При МАКШ вмешательство, как правило, включало маммарокоронарное шунтирование (МКШ) или биМКШ коронарных артерий передней и боковой стенок левого желудочка и АКШ остальных коронарных артерий. Гибридный подход и параллельное трансфеморальное экстракорпоральное кровообращение без кардиоплегии были допустимы для достижения требующегося уровня реваскуляризации. Как правило, гибридная реконструкция заключалась в комбинации полной артериальной реваскуляризации системы ЛКА и ЧКВ в бассейне правой коронарной артерии.

Предоперационная характеристика пациентов по основным клиническим параметрам представлена в табл. 1. Возраст пациентов составил $58,5 \pm$ 7,9 года, в группе было 70 (14,0\%) женщин. Сахарный диабет выявлен у 105 (21,0\%) пациентов, ожирение - у 187 (37,4\%), генерализованный атеросклероз - у 121 (24,2\%) пациента. Риск по EuroSCORE составил 1,2 $\pm 0,7$, степень поражения коронарного русла по SYNTAX Score - 26,4 8,8.

Данные выражались как числовые значения (проценты) $-n(\%)$, среднее значение \pm стандартное отклонение $(M \pm \sigma)$, медиана и интерквартильный размах - Me (LQ; UQ). Анализ распределения значений показателей на соответствие закону нормального распределения проводили с помощью теста Шапиро-Уилка. Свобода от основных неблагоприятных сердечных и мозговых событий в среднесрочном отдаленном периоде наблюдения оценивалась с помощью процедуры Каплана-Майера. При изучении непосредственных результатов лечения основными конечными точками исследования стали смерть от любой причины, нефатальные острый инфаркт миокарда и инсульт до выписки из стационара или в течение 30 дней после выполнения КШ. Вторичные конечные точки исследования включали кровопотерю во время операции и в первые сутки после вмешательства, частоту гемотрансфузий, впервые возникшую почечную недостаточность и раневые осложнения. При изучении среднесрочных отдаленных результатов конечными точками исследования были основные неблагоприятные сердечные и мозговые события.

Инсульт определен как быстрое развитие стойкой очаговой неврологической симптоматики, случившееся интраоперационно или в послеоперационном периоде. Генерализованный атеросклероз определен как наличие у пациента с ИБС атеросклероза артерий нижних конечностей или атеросклероза артерий, питающих головной мозг, с гемодинамически значимым поражением (стеноз $>50 \%$ ). Ожирение определено как наличие ИМТ $\geq 30 \mathrm{kr} / \mathrm{m}^{2}$. Впервые возникшая почечная недостаточность определена как необходимость во временном или постоянном использовании любого вида гемодиализа после операции. Поверхностная раневая инфекция определена как санация гнойно-воспалительного процесса мягких тканей. Глубокая раневая инфекция определена как санация гнойно-воспалительного процесса грудины, ребер, средостения или плевральной полости. Стерномедиастинит определен как инфекционное осложнение, возникшее после рассечения грудины,

Таблица 1. Предоперационная характеристика пациентов $(n=500)$ по основным клиническим параметрам

\begin{tabular}{|l|c|}
\hline \multicolumn{1}{|c|}{ Показатель } & 3начение \\
\hline Возраст, годы & $58,5 \pm 7,9$ \\
\hline Женский пол & $28,7 \pm 4,1$ \\
\hline Индекс массы тела, кг/м² & $55,9 \pm 7,3$ \\
\hline Фракция выброса левого желудочка, \% & $187(37,4)$ \\
\hline Ожирение & $123,2 \pm 36,5$ \\
\hline Конечный диастолический объем левого желудочка, мл & $97(19,4)$ \\
\hline Стеноз ствола левой коронарной артерии & $121(24,2)$ \\
\hline Генерализованный атеросклероз & $105(21,0)$ \\
\hline Сахарный диабет & $144,9 \pm 12,6$ \\
\hline Гемоглобин крови, г/л & $0,95 \pm 0,18$ \\
\hline Креатинин крови, мг \% & $1,2 \pm 0,7$ \\
\hline EuroSСОRE II & $26,4 \pm 8,8$ \\
\hline SYNTAX Sсоге & \\
\hline
\end{tabular}

Примечание. Здесь и в табл. 2: данные представлены в виде $n(\%) ; M \pm \sigma ; M e(L Q ; U Q)$. 
с вовлечением в инфекционный процесс костной ткани грудины, тканей средостения, с вовлечением/без в процесс поверхностных мягких тканей, с наличием/без стабильности грудины. Послеоперационная пневмония определена как наличие воспалительной инфильтрации легочной ткани при рентгенологическом исследовании в послеоперационном периоде, связанной с инфекционным воспалением и/или гипостатическими явлениями в легких. Плевральная эффузия определена как скопление жидкости между слоями плевры в послеоперационном периоде, которое потребовало хотя бы одной пункции плевральной полости для ее удаления. Основные неблагоприятные сердечные и мозговые события (ОНСМС) определены как смерть от сердечно-сосудистой причины, нефатальный острый инфаркт миокарда, нефатальный инсульт или повторная реваскуляризация миокарда. Гемотрансфузия определена как переливание любых компонентов крови во время и после операции до выписки из стационара. За единицу или дозу цельной крови принят объем 450 мл. В случае компонентов крови 1 единица представляет собой такое количество соответствующей субстанции, которое обычно содержится в 1 единице (дозе) цельной крови. Так, за 1 единицу эритроцитной массы принят объем 300 мл, за 1 единицу свежезамороженной плазмы - 200 мл. Время достижения полной физической активности определено как способность пациента совершать прогулки по $\geq 30$ мин ежедневно и свободно работать верхней половиной туловища в повседневной жизни без ограничений. Экстренная конверсия определена как переход к ЭКК при операции на работающем сердце при стойких гемодинамических нарушениях, резистентных к медикаментозной и электроимпульсной терапии.

\section{Результаты}

Характеристика периода операции и раннего послеоперационного периода представлена в табл. 2.

Средняя продолжительность операции составила 254,5 84,7 мин, число дистальных анастомо-

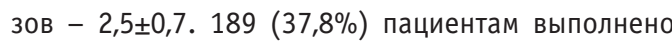
МАКШ, при котором, как правило, использовались одна или обе внутренние грудные артерии in situ в сочетании с аутовенозными графтами от восходящей аорты. КШ без манипуляций на аорте

Таблица 2. Характеристика периода операции и раннего послеоперационного периода у пациентов $(n=500)$

\begin{tabular}{|c|c|}
\hline Показатель & Значение \\
\hline Продолжительность операции, мин & $254,5 \pm 84,7$ \\
\hline Маммароаортокоронарное шунтирование & $189(37,8)$ \\
\hline Коронарное шунтирование без манипуляций на аорте & $311(62,2)$ \\
\hline в том числе гибридная реваскуляризация миокарда & $29(5,8)$ \\
\hline Полная артериальная реваскуляризация миокарда & $276(55,2)$ \\
\hline Количество дистальных анастомозов & $2,5 \pm 0,7$ \\
\hline Переход к ЭКК & $24(4,8)$ \\
\hline в том числе экстренная конверсия к ЭКК & $13(2,6)$ \\
\hline Переход на стернотомию & $4(0,8)$ \\
\hline Интраоперационная кровопотеря, мл & $250(200 ; 300)$ \\
\hline Кровопотеря в 1-е сутки после операции, мл & $270(150 ; 350)$ \\
\hline Трансфузия крови и ее компонентов & $47(9,4)$ \\
\hline Длительность послеоперационной ИВЛ, ч & $5,0(3,0 ; 8,0)$ \\
\hline Послеоперационная ИВЛ >24 ч & $4(0,8)$ \\
\hline Длительность пребывания в ОРИТ, ч & $20,0(17,0 ; 24,0)$ \\
\hline Впервые возникшая мерцательная аритмия & $31(6,2)$ \\
\hline Поверхностная раневая инфекция грудной клетки & $11(2,2)$ \\
\hline Глубокая раневая инфекция грудной клетки & $1(0,2)$ \\
\hline Послеоперационная пневмония & $38(7,6)$ \\
\hline Послеоперационное кровотечение с повторной операцией & $2(0,4)$ \\
\hline Плевральная эффузия & $49(9,8)$ \\
\hline Впервые возникшая почечная недостаточность & $0(0,0)$ \\
\hline Делирий & $9(1,8)$ \\
\hline Инсульт & $2(0,4)$ \\
\hline Инфаркт миокарда & $7(1,4)$ \\
\hline 30-дневная летальность & $3(0,6)$ \\
\hline Длительность послеоперационного пребывания в стационаре, дни & $7(7 ; 10)$ \\
\hline
\end{tabular}

Примечание. ИВЛ - искусственная вентиляция легких; ОРИТ - отделение реанимации и интенсивной терапии; ЭКК - экстракорпоральное кровообращение. 


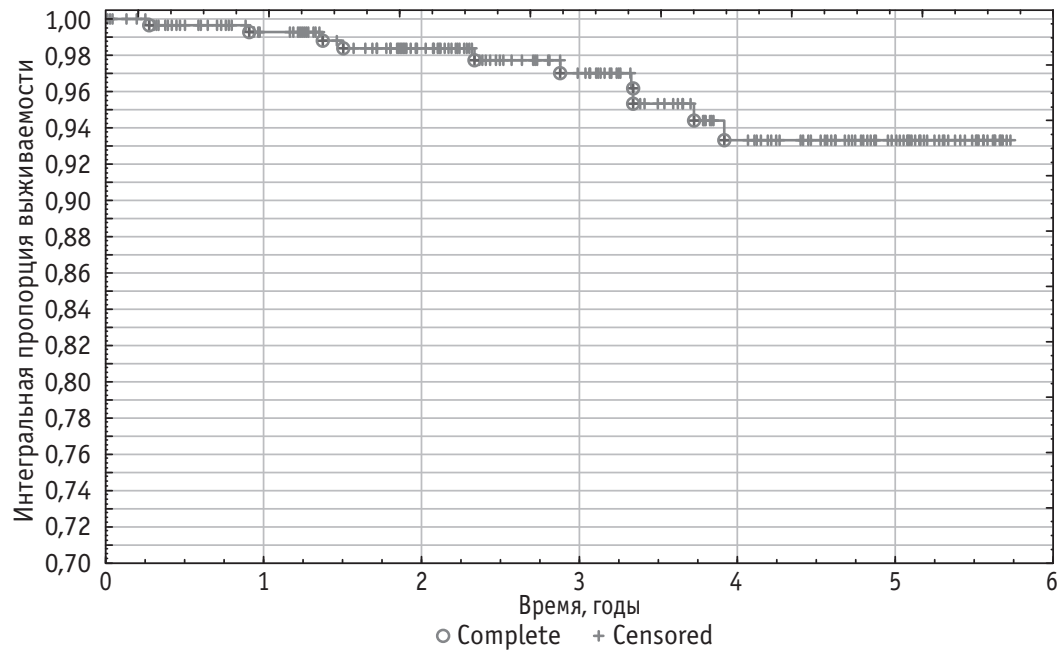

Рис. 1. Кумулятивная общая выживаемость в отдаленном периоде наблюдения с учетом сердечно-сосудистых и других причин

Fig. 1. Cumulative overall survival in the remote observation period, taking into account cardiovascular and other reasons произведено 311 (62,2\%) пациентам, в том числе полная артериальная реваскуляризация миокарда 276 (55,2\%) пациентам, гибридная реваскуляризация миокарда - 29 (5,8\%) пациентам. Левую внутреннюю грудную артерию применили у 494 (98,8\%) пациентов, правую внутреннюю грудную артерию - у 120 (24,0\%), лучевую артерию у 173 (34,6\%), аутовену - у 195 (39,0\%), лапароскопически выделенную правую желудочно-сальниковую артерию - у 4 (0,8\%) пациентов.

ЭКК применено у 24 (4,8\%) пациентов, из них экстренная конверсия выполнена у 13 (2,6\%) пациентов. Переход на стернотомию осуществлен у 4 (0,8\%) пациентов. Основной причиной экстренных конверсий к ЭКК и стернотомии были тяжелые желудочковые аритмии с коллапсом гемодинамики, не поддающиеся комплексной коррекции.

Медиана интраоперационной кровопотери составила $250(200 ; 300)$ мл, кровопотери за 1-е сутки после вмешательства - 270 (150; 350) мл. Переливание крови и ее компонентов произведено у $47(9,4 \%)$ пациентов.

Длительность искусственной вентиляции легких в послеоперационном периоде составила 5,0 $(3,0 ; 8,0)$ 4, время нахождения в отделении реанимации и интенсивной терапии - 19,5 (17,0; $24,0)$ ч. Необходимость продленной искусственной вентиляции легких >24 ч возникла у 4 (0,8\%) пациентов.

В структуре осложнений превалировали мерцательная аритмия - 6,2\% (31 пациент), послеоперационная пневмония - 7,6\% (38 пациентов) и плевральная эффузия - 9,8\% (49 пациентов). Частота повторных операций по поводу послеоперационного кровотечения составила 0,4\% (2 пациента). Развитие поверхностной раневой инфекции грудной клетки отмечено у 11 (2,2\%) пациентов. В то же время при МИКШ не наблюдалось случаев инфекции со стороны средостения, ребер, грудины и плевральной полости. Периоперационный ин- сульт зафиксирован у 2 (0,4\%) пациентов, инфаркт миокарда - у 7 (1,4\%), 30-дневная летальность составила 0,6\% (3 пациента).

Длительность послеоперационного пребывания в стационаре составила $7(7 ; 10)$ койко-дней. Медиана времени возврата к полной физической активности составила $14(9 ; 24)$ дней.

Медиана длительности наблюдения составила $4,8(1,4 ; 7,3)$ года. В отдаленном периоде после МИКШ от сердечно-сосудистых причин умерли 6 пациентов, от других причин - 4 пациента. На рис. 1 отражена выживаемость в отдаленном периоде наблюдения с учетом сердечно-сосудистых и других причин. Кумулятивная выживаемость после мини-инвазивного КШ, рассчитанная по методу Каплана-Майера, без учета госпитальной летальности составила через 1 год - 99,1\%, 2 года - 98,2\%, 3 года - 97\%, 4 года - 93,4\%, 5 лет - 93,4\%.

В отдаленном периоде наблюдения 3 пациента перенесли нефатальный инфаркт миокарда с ЧКВ, 4 пациента - нефатальный инсульт и 4 пациентам выполнена повторная реваскуляризация миокарда из-за рецидива стенокардии. Из них 2 пациентам выполнено повторное КШ задней межжелудочковой ветви правой коронарной артерии правой желудочно-сальниковой артерией из мини-лапаротомии и 2 пациентам - ЧКВ. На рис. 2 представлены данные по отсутствию основных неблагоприятных сердечных и мозговых событий в отдаленном периоде наблюдения. Кумулятивный показатель отсутствия ОНСМС в отдаленном периоде после МИКШ составил через 1 год - 97,5\%, 2 года - 95,3\%, 3 года - 94\%, 4 года - 92\%, 5 лет - 90,7\%.

\section{Обсуждение}

В соответствии с полученными данными непосредственные результаты МИКШ не уступают, а по ряду показателей отличаются в лучшую сторону от таковых при традиционном КШ на работающем 
Рис. 2. Свобода от основных неблагоприятных сердечных и мозговых событий в отдаленном периоде наблюдения

Fig. 2. Freedom from major adverse cardiac and cerebrovascular events in a remote observation period

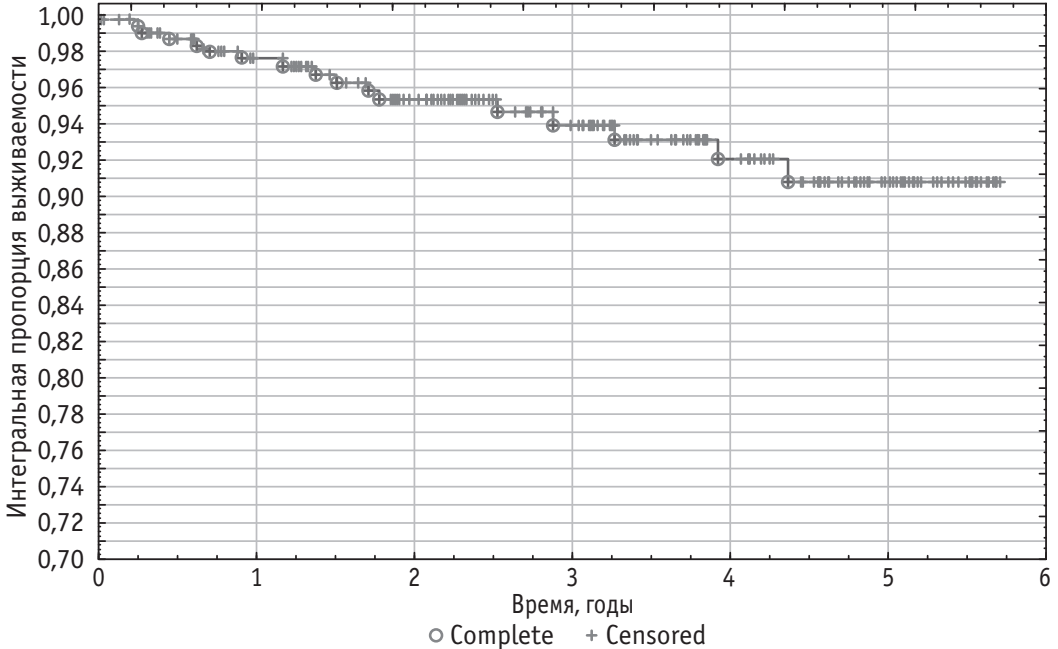

сердце или с ЭКК. Это согласуется с выводами недавно опубликованных исследований в отношении непосредственных результатов МИКШ [10-12]. По всей видимости, ЭКК и стернотомия являются важными факторами, влияющими на объем периоперационной кровопотери, частоту и объем гемотрансфузий, а также на развитие глубокой раневой инфекции. Более длительное время возврата к полной физической активности при традиционном АКШ в первую очередь связано с длительным ограничением физических нагрузок на верхнюю половину туловища после стернотомии.

На сегодняшний день имеются единичные сообщения об отдаленных результатах многососудистого мини-АКШ. J.T. McGinn и соавт. представили исследование по оценке выживаемости и свободы от основных неблагоприятных событий со средней

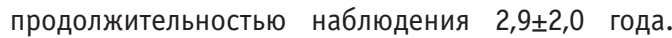
Было установлено, что мини-АКШ - безопасное и воспроизводимое вмешательство с выживаемостью в отдаленном периоде наблюдения $96,1 \pm 0,9 \%$ и частотой повторной реваскуляризации миокарда 5,1\% в виде ЧКВ [13]. Е.A. Barsoum и соавт. сообщили, что в 5-летнем периоде наблюдения в группе пациентов в возрасте $\geq 75$ лет после мини-АКШ общая смертность была достоверно ниже по сравнению с группой пациентов после КШ из стернотомии (19,7 и 47,7\% соответственно) [14].

Данное исследование позволило сделать основное заключение, что мини-инвазивная реваскуляризация миокарда может быть безопасно использована у пациентов с ИБС, которым показано многососудистое КШ в плановом порядке, с сохранением клинической эффективности КШ в среднесрочном отдаленном периоде наблюдения. Это подтверждается низким уровнем смертности, частоты развития нефатального инфаркта миокарда, инсульта и повторной реваскуляризации миокарда в отдаленные сроки наблюдения после выполнения МИКШ.
За счет снижения частоты периоперационных осложнений и уменьшения длительности нахождения в стационаре применение мини-инвазивных вмешательств может значительно увеличить удовлетворенность пациента качеством лечения. Немаловажную роль при этом имеет потенциальная возможность ускорения возврата к полной физической активности и трудовой деятельности. Ряд пациентов важное значение придают косметическому эффекту. Однако на одно из ведущих мест следует поставить психологический фактор, благодаря которому пациенты в случае необходимости хирургической реваскуляризации миокарда практически также охотно соглашаются на МИКШ, как и на ЧКВ, в отличие от традиционного АКШ.

В то же время большинство операций КШ на современном этапе выполняются с ЭКК, кардиоплегией, использованием аутовенозных графтов, через тотальную срединную стернотомию. Ограниченное применение многососудистого МИКШ в первую очередь связано с мнением многих хирургов, что данные вмешательства сопряжены с высокой технической сложностью и требуют длительного периода обучения. По мнению S. Head и соавт. [15], несмотря на видимые преимущества, освоение новых технологий остается на низком уровне вследствие: а) привычки хирургов оперировать пациентов традиционными методами, нежелания перемен и отсутствия готовности прохождения периода обучения, свойственного новой технологии; б) необходимости более серьезных требований к техническим навыкам хирурга; в) потенциальных сложностей, связанных с освоением новых методов и устройств; г) возможного увеличения временных затрат; д) логи стических вопросов, связанных с необходимостью приобретения нового оборудования и инструментов, а также с изменением планирования и режима 
работы. Эти факторы играют основную роль в сохранении имеющихся протоколов, особенно если предполагаемые преимущества новых методов пока четко не доказаны.

D. Une и соавт. на основании опыта лечения 210 пациентов сообщили, что внедрение МИКШ может быть проведено с низким уровнем периоперационного риска. При этом ЭКК использовалось в 24,3\% и конверсия к стернотомии - в 4,8\% случаев [16]. Безопасная инициализация технологии МИРМ согласуется с результатами нашего исследования с включением начального опыта, при этом переход к ЭКК и стернотомии осуществлялся еще реже - в 4,8 и 0,8\% случаев соответственно.

Также надо учитывать, что некоторые технологии, например тотально эндоскопическое или робот-ассистированное КШ, вследствие своей трудоемкости будут оставаться уделом высокоспециализированных центров. Что касается МИКШ, рассматриваемая операция не требует дорогостоящего оборудования, выполняется под прямым контролем зрения и, как правило, стандартными коронарными инструментами. При этом вмешательство является универсальным - могут быть использованы техника без манипуляций на аорте, бимаммарное шунтирование, КШ с проксимальными анастомозами с восходящей аор-

\section{Литература/References}

1. Lapierre H., Chan V., Sohmer B., Mesana T.G., Ruel M. Minimally invasive coronary artery bypass grafting via a small thoracotomy versus off-pump: a casematched study. Eur J Cardiothorac Surg. 2011; 40: 80410. doi: https://doi.org/10.1016/j.ejcts.2011.01.066

2. Ruel M., Une D., Bonatti J., McGinn J.T Jr. Minimally invasive coronary artery bypass grafting: is it time for the robot? Curr Opin Cardiol. 2013; 28 (6): 639-45. DOI: https://doi.org/10.1097/HCO.0b013e3 283653fd1

3. Poston R.S., Tran R., Collins M., Reynolds M., Connerney I., Reicher B., et al. Comparison of economic and patient outcomes with minimally invasive versus traditional off-pump coronary artery bypass grafting techniques. Ann Surg. 2008; 248: 638-46. DOI: https://doi. org/10.1097/SLA.0b013e31818a15b5

4. Puskas J.D., Williams W.H., Mahoney E.M., Huber P.R., Block P.C., Duke P.G., et al. Off-pump vs conventional coronary artery bypass grafting: early and 1-year graft patency, cost, and quality-of-life outcomes: a randomized trial. JAMA. 2004; 291 (15): 1841-9. DOI: https://doi.org/10.1001/jama.291.15.1841

5. Cohen D.J., Van Hout B., Serruys P.W., Mohr F.W., Macaya C., den Heijer P., et al. Quality of life after PCl with drug-eluting stents or coronary-artery bypass surgery. N Engl J Med. 2011; 364 (11): 1016-26. DOl: https:// doi.org/10.1056/NEJMoa1001508 той, при необходимости - вспомогательное кровообращение и гибридный подход в сочетании с ЧКВ.

В заключение отметим: несмотря на кажущуюся техническую сложность многососудистого МИКШ, с каждым годом все больше хирургов в нашей стране осваивают данную технологию, что говорит о ее воспроизводимости. Необходимость отбора пациентов является важным ограничивающим фактором в настоящее время, однако методика МИКШ все еще находится в ранней фазе своего развития, по мере ее распространения и накопления хирургического опыта критерии отбора могут расширяться.

\section{Заключение}

мИКШ является безопасным вмешательством, ассоциируясь с низкой частотой периоперационных осложнений, конверсий к ЭКК и стернотомии, короткими длительностью пребывания пациента в стационаре и временем возврата к полной физической активности.

МИКШ может быть применено у пациентов с ИБС при многососудистом поражении с сохранением эффективности КШ в среднесрочном отдаленном периоде наблюдения, сравнимом с традиционным АКШ.

6. Serruys P.W., Morice M.C., Kappetein A.P., Colombo A., Holmes D.R., Mack M.J., et al. Percutaneous coronary intervention versus coronary-artery bypass grafting for severe coronary artery disease. N Engl J Med. 2009; 360 (10): 961-72. DOI: https://doi.org/10.1056/NEJMoa0804626

7. Shennib H. Evolving strategies in minimally invasive coronary artery surgery. Int J Cardiol. 1997; 62 (1): 81-8.

8. Calafiore A.M., De Giammarco G., Teodori G., Bosco G., D’Annunzio E., Barsotti A., et al. Left anterior descending coronary artery grafting via left anterior small thoracotomy without cardiopulmonary bypass. Ann Thorac Surg. 1996; 61 (6): 1658-63.

9. Ziankou A.A., Ostrovsky Y.P., Laiko M.G., Vykhrystsenka K.S., Chuyashou V.A., Zhyhalkovich A.S. Hospital and mid-term results of prospective randomized controlled trial MICSREVS - Minimally Invasive Cardiac Surgery REVascularization Strategy [Electronic resource]. In: 30th EACTS Annual Meeting, Barcelona, Spain, 1-5 October 2016. URL: http://medialibrary.eacts.cyim. com/mediatheque/media. aspx?mediald=18441\&chann el=10233 (date of access November 20, 2016)

10. Lemma M., Atanasiou T., Contino M. Minimally invasive cardiac surgery-coronary artery bypass graft [Electronic resource]. Multimed Man Cardio-Thoracic Surg. 2013. URL: http://mmcts.oxfordjournals.org/content/2013/mmt007. full (date of access November 2, 2017) 
11. McGinn J.T. Jr, Usman S., Lapierre H., Pothula V.R., Mesana T.G., Ruel M. Minimally invasive coronary artery bypass grafting: dual center experience in 450 consecutive patients. Circulation. 2009; 120 (11 Suppl): 78-84. DOI: https://doi.org/10.1161/CIRCULATIONAHA.108.840041

12. Nambiar P., Mittal C. Minimally invasive coronary bypass using internal thoracic arteries via a left minithoracotomy "the Nambiar Technique". Innovations (Phila). 2013; 8 (6): 420-6. DOI: https://doi.org/10.1097/ IMI.0000000000000035

13. McGinn Jr J.T., Shariff M.A., Nabagiez J.P., CarIuchi R., Sadel S., Sharma R., et al. Minimally invasive CABG is safe and reproducible: report on the first thousand cases. 14th ISMICS Annual Scientific Meeting; 2014 May 28-31; Boston, USA. Innovations. 2014; 9 (3): 158.
14. Barsoum E.A., Azab B., Shah N., Patel N., Shariff M.A., Lafferty J., et al. Long-term mortality in minimally invasive compared with sternotomy coronary artery bypass surgery in the geriatric population (75 years and older patients). Eur J Cardiothorac Surg. 2015; 47 (5): 862-7. DOI: https://doi.org/10.1093/ejcts/ezu267

15. Head S.J., Börgermann J., Osnabrugge R.L.J., Kieser T.M., Falk V., Taggart D.P., et al. Coronary artery bypass grafting: part 2 - optimizing outcomes and future prospects. Eur Heart J. 2013; 34 (37): 2873-86. DOI: https://doi.org/10.1093/eurheartj/eht284

16. Une D., Lapierre H., Sohmer B., Rai V., Ruel M. Can minimally invasive coronary artery bypass grafting be initiated and practiced safely?: a learning curve analysis. Innovations (Phila). 2013; 8 (6): 403-9. DOI: https://doi. org/10.1097/IMI.0000000000000019 